\title{
TYPE TRANSITIONS IN STARBURSTS POWERED AGN
}

\author{
ITZIAR ARETXAGA ${ }^{1}$ and ROBERTO TERLEVICH ${ }^{2}$ \\ ${ }^{1}$ Dpto. de Física Teórica, C-XI. Universidad Autónoma de Madrid. Cantoblanco 28049 Madrid. \\ Spain. \\ ${ }^{2}$ Royal Greenwich Observatory. Madingley Road, CB3 OEZ Cambridge. U.K.
}

There is mounting evidence that type transitions are a common property among Active Galactic Nuclei: the broad lines in, at least, eleven Seyfert galaxies have ocassionaly appeared and subsequently disappeared leading to the reclasification of their nuclei from type 1-1.5 to type 1.8-2 or viceversa.

Type transitions are an outstanding property in the evolution of high metallicity massive starbursts due to the stochastic nature of the supernova explosions that take place in them. In the Starburst theory, the variability observed in radio quiet AGN is thought to be produced by the supernova (SN) and the compact supernova remnant (cSNR) activity of such a cluster (Terlevich et al. 1992, MNRAS, 255, 713). According to the theory, the luminosity of these systems is directly related to its SN rate (Aretxaga \& Terlevich 1993, Astrophys. Spa. Sci., 205, 69). For low and medium luminosity AGN ( $\left.\overline{M_{\mathrm{B}}} \lesssim-22.5 \mathrm{mag}\right)$, the low SN rates derived $\left(\nu_{\mathrm{SN}} \lesssim 1 \mathrm{yr}^{-1}\right)$ suggests a non negligible probability that the broad components of the emission lines are undetectable at some epoch. The stochastic nature of the SN explosions allows to obtain periods of time in which there is a lack of new explosions, while the existing remnants are too old to produce broad lines.

The AGN in which activity transitions have been observed are all below the predicted luminosity cutoff $\left(\overline{M_{B}} \approx-22.5 \mathrm{mag}\right)$, as can be seen in Figure 1.

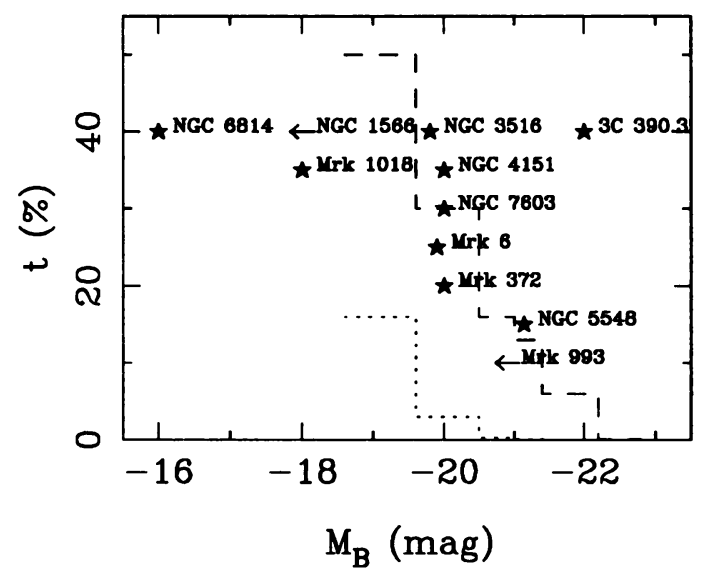

Fig. 1. Fraction of time spent as Seyfert of type 1.9 or 2 by theoretical stellar clusters of a given mean luminosity: the dashed line corresponds to type 1.9 stages and the dotted line to type 2 stages. The symbols represent the luminosities of the AGN known to have experienced type transitions: the stars indicate mean luminosities and the arrows upper limits to mean luminosities.

T. J.-L. Courvoisier and A. Blecha: Multi-Wavelength Continuum Emission of AGN, 438.

(c) 1994 IAU. Printed in the Netherlands. 